\title{
Mycobacterium tuberculosis/Cryptococcus neoformans Co-Infection in an Immunocompetent Non-HIV Patient
}

\author{
Salma Albahrani', Donya Al Hassan², Yusuf Vapra ${ }^{3}$, Saad Alsubaiae ${ }^{4}$ and Amal Shilash ${ }^{5, *}$ \\ ${ }^{1}$ Department of Internal Medicine, Infectious disease section, King Fahd Military Medical complex, Dhahran, Eastern Province, Kingdom of \\ Saudi Arabia \\ ${ }^{2}$ Department of Radiology, Cardiothoracic section, King Fahd Military Medical complex, Dhahran, Eastern Province, Kingdom of Saudi Arabia \\ ${ }^{3}$ Department of Internal Medicine, Pulmonology section, King Fahd Military Medical complex, Dhahran, Eastern Province-Kingdom of Saudi Arabia \\ ${ }^{4}$ Department of Histopathology, King Fahd Military Medical complex, Dhahran, Eastern Province, Kingdom of Saudi Arabia \\ ${ }^{5}$ Department of Infection Control, King Fahd Military Medical complex, Dhahran, Eastern Province, Kingdom of Saudi Arabia
}

"Corresponding author: Amal Shilash, Department of Infection Control, King Fahd Military Medical complex, Dhahran 31952, Eastern Province, Kingdom of Saudi Arabia, Tel: 00966509986630; E-mail: amalsalshammari@gmail.com

Received: 21 May, 2020 | Accepted: 17 Jun, 2020 | Published: 24 Jun, 2020

Citation: Albahrani S, Al Hassan D, Vapra Y, Alsubaiae S, Shilash A (2020) Mycobacterium tuberculosis/Cryptococcus neoformans Co-Infection in an Immunocompetent Non-HIV Patient. Autoimmun Infec Dis 3(2): dx.doi.org/10.16966/2470-1025.127

Copyright: (c) 2020 Albahrani S, et al. This is an open-access article distributed under the terms of the Creative Commons Attribution License, which permits unrestricted use, distribution, and reproduction in any medium, provided the original author and source are credited.

\begin{abstract}
Background: Tuberculosis and cryptococcosis co-infection usually occurs in immunocompromised patients with impaired cell-mediated immunity. Here, we report a case of disseminated tuberculosis with co-existing pulmonary cryptococcosis in a non-HIV patient. However, there is a history of preceding exposure to anti-tumor necrosis agents.

Methods: The method consists of a retrospective descriptive analysis of a single case as a case report.

Results: A 62-year-old Saudi female presented to the Emergency Department (ED) with complaints regarding the two-month history of appetite loss and shortness of breath. Her past medical history showed a history of receiving anti-tumor necrosis factor for Psoriasis nine months before the onset of the symptoms. The chest Computed Tomography (CT) showed bilateral diffuse micro-nodules in the lungs. Therefore, Lung nodule biopsy was done and showed epithelioid cell granuloma and fungal elements of yeast. Positive staining for both Periodic Acid-Schiff (PAS) stain and Gomori Methenamine Silver (GMS) stain. The latter is suggestive of Histoplasmosis which is not endemic in Saudi Arabia. The molecular identification came back as Cryptococcus neoformans. Furthermore, bronchoalveolar lavage was done and a follow-up test with polymerase chain reaction showed Mycobacterium tuberculosis. During her course of admission, the patient had abnormal myoclonic movements. Brain MRI showed ring-enhancing lesions in bilateral basal ganglia. Moreover, the cryptococcal antigen was negative in Cerebral Spinal Fluid (CSF) and positive in serum testing. According to these findings, disseminated tuberculosis in the lung and brain was suspected with pulmonary cryptococcosis where antimycobacterial therapy and anti-fungal were initiated. After two months in therapy, the patient improved.

Conclusion: Clinicians should be aware that tuberculosis co-infection with cryptococcosis can co-exist in non-HIV patients without underlying diseases, although it is rare.
\end{abstract}

\section{Introduction}

Tuberculosis and cryptococcosis co-infection usually, albeit uncommonly occurs in immunosuppressed patients with impaired cell-mediated immunity or HIV-positive patients [1]. However, there are few reported cases about such co-infection in non-HIV patients without underlying diseases [1]. Here we report a case of disseminated tuberculosis with co-existing pulmonary Cryptococcosis in an immunocompetent patient. The diagnosis was concluded based on bronchoalveolar lavage and brain abscesses following lung biopsy for Cryptococcus neoformans. There is evidence that both Mycobacterium tuberculosis and Cryptococcus neoformans may have suppressive effects on the host immune system which might explain the coinfection in healthy individuals [2].

This case report expands our standing of these infections in an individual with a history of treatment by anti-TNF nine months prior to the clinical presentation.

\section{Case Presentation}

A 62-year-old Saudi female presented to the emergency department (ED) complaining of a two-month history of loss of appetite, weight loss of about ten kilograms, and periodic subjective fever. Also, she gave a history of shortness of breath and productive cough of whitish 
sputum. She is not a smoker and unaware of neither previous diagnosis with tuberculosis nor any contact with confirmed tuberculosis cases. Medication history showed she is currently on escitalopram for depression and history of Adalimumab use for psoriasis; her last dose nine months ago which she was on for one year. The patient denied any corticosteroid use and unaware if she has been screened for tuberculosis before. She is living in the South (Fifa beside Jizan in Saudi Arabia). She has been raising a lot of cats; and has had no contact with birds, pigeon roosts, or chicken-coop-cleaning; there are no nearby building construction or demolitions, and she never traveled outside the kingdom. Physical examination: revealed a pale-looking elderly cachectic with Parkinson's disease feature. Chest examination showed bilateral upper lobe crackles with decreased air entry on the right side. Initial laboratory works showed a normal range of white blood cell count (WBC), no anemia with normal hemoglobin value, normal renal functions with normal value of blood urea nitrogen (BUN), and creatinine. Normal value of albumin-Human immunodeficiency virus (HIV) $\mathrm{Ag} / \mathrm{Ab}$ combo nonreactive $\mathrm{HBsAg}$ nonreactive, hepatitis $\mathrm{C}$ virus $\mathrm{Ab}$ Nonreactive, C-reactive protein (CRP)12, antinuclear antibodies (ANA) at $<1: 80$, hypercalcemia with calcium level at 2.60 $\mathrm{mmol} / \mathrm{l}$ (2.12-2.252). Radiologically, Chest X-rays showed a moderate amount of pleural effusion in the right lung with right lower lobe consolidations and nodular opacities in the right middle zone. Hence, a chest computed tomography (CT) scan showed bilateral pleural effusions with associated bilateral basal pulmonary nodules and background of interstitial lung disease and bilateral bronchiectasis changes affecting the posterior segment of its lower lobes as well as in the right middle lung lobe. Furthermore, the abdomen CT scan shows omental thickening anterior to the transverse colon and multiple splenic focal lesions and few para-aortic subcentimetric lymph nodes as per figures 1-4.

Furthermore, Pleural fluid aspiration was performed. The fluid was found to be exudate, and the bacterial culture is negative. An AcidFast Bacilli (AFB) smear is negative, and an MTB/Rifampicin gene expert and culture are requested. A thoracic guided biopsy was done, and the standard four-regimen therapy was started before the results because of tuberculosis are endemic in the city where the patient lives. One week into the admission, the patient's fever improved. On General appearance, the patient had a mask-like face and myoclonic lower limb movements. Neurological examination showed no obvious cranial nerve abnormality apart from slow, saccadic eye movement. The motor examination showed an increase in tone for both upper limbs, power $4 / 5$ all muscle groups. Lead pipe rigidity with normal power and left up going planter, sustained ankle clonus bilaterally, gait, and coordination were difficult to be assessed. Further imaging of the brain was ordered and the brain CT showed diffuse increased periventricular T2 hyperintensities with multiple discrete, confluent, non-enhancing variable-sized signal abnormalities and a few small ring-enhancing lesions are seen in the bilateral basal ganglia, and the right centrum and left temporal cortical enhancing focus was noted as well as per figure 5. Lung biopsy was positive for granuloma and fungal elements look like yeast and negative for culture. Positive staining for both Periodic Acid-Schiff (PAS) stain and Gomori Methenamine Silver (GMS) stain. The latter is suggestive of histoplasmosis as per figures 6-8.

The patient started treatment with anti-fungal agents after lung biopsy, regardless of the impression of histoplasmosis that was not endemic in Saudi Arabia. The choice for liposomal amphotericin $5 \mathrm{mg} / \mathrm{kg}$ this was chosen based on the brain lesions, and the azoles drugs were avoided because of hepatotoxicity of the anti-TB agents that the patient exhibited. However, during treatment, the patient exhibited mild improvement only and showed drug-induced elevated liver enzymes with a mixed pattern. Therefore, the anti-TB medications were discontinued. Later, the bronchoscopy was repeated and showed positive MTB/RIF PCR gene expert and AFB smear and culture resulted as positive for Cryptococcus neoformans. Further stating with mucicarmine stain that showed clear encapsulated yeast. Antituberculosis regimens (isoniazid, rifampicin, ethambutol, and moxifloxacin) were resumed gradually and with more caution this time. Dexamethasone was added, and step down therapy of fluconazole was initiated. Also, a lumbar puncture was performed to assess the cerebrospinal fluid (CSF) and the AFB smear and culture. The MTB/ RIF PCR was negative, and the India ink stain test was negative. The

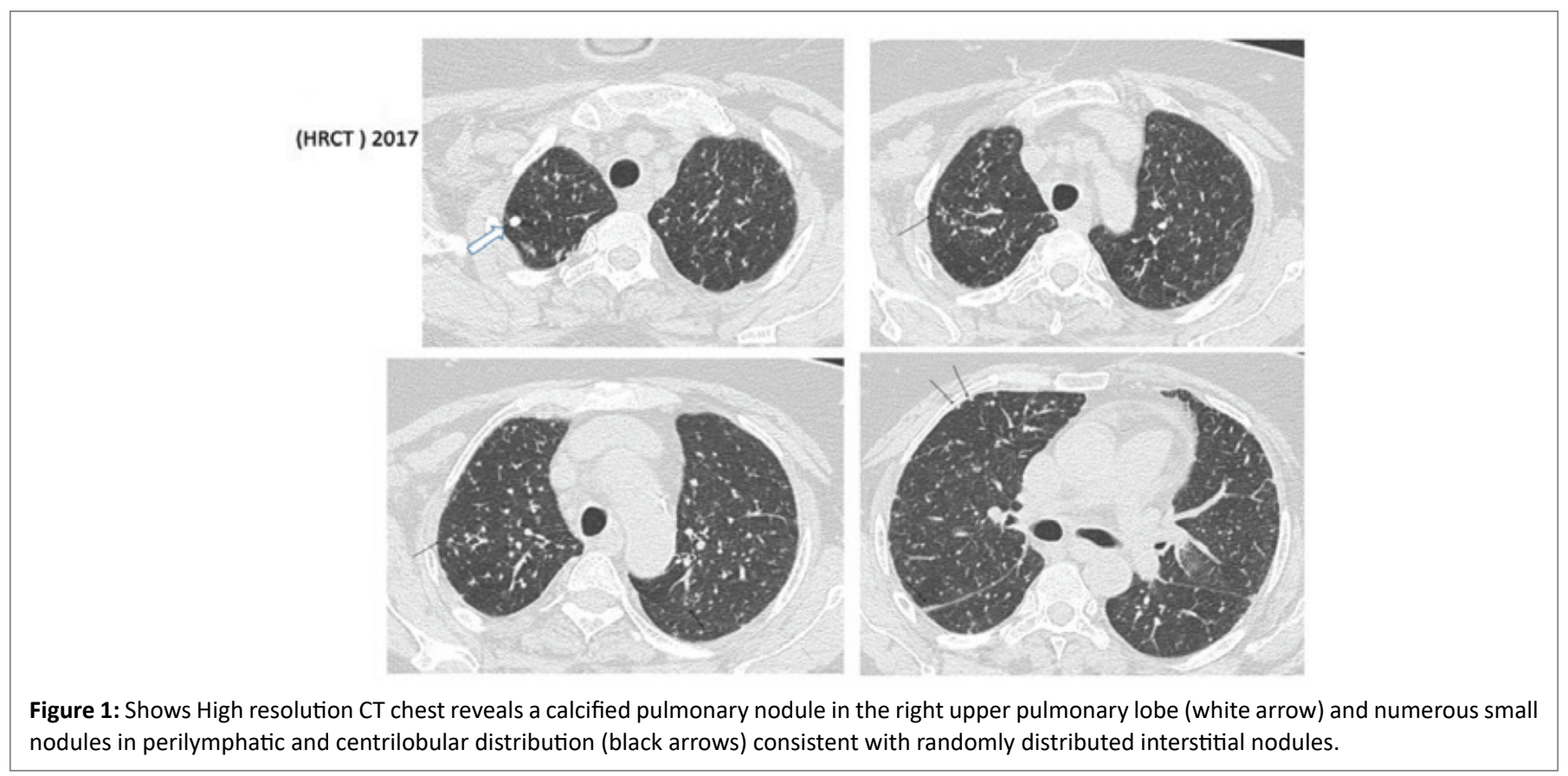



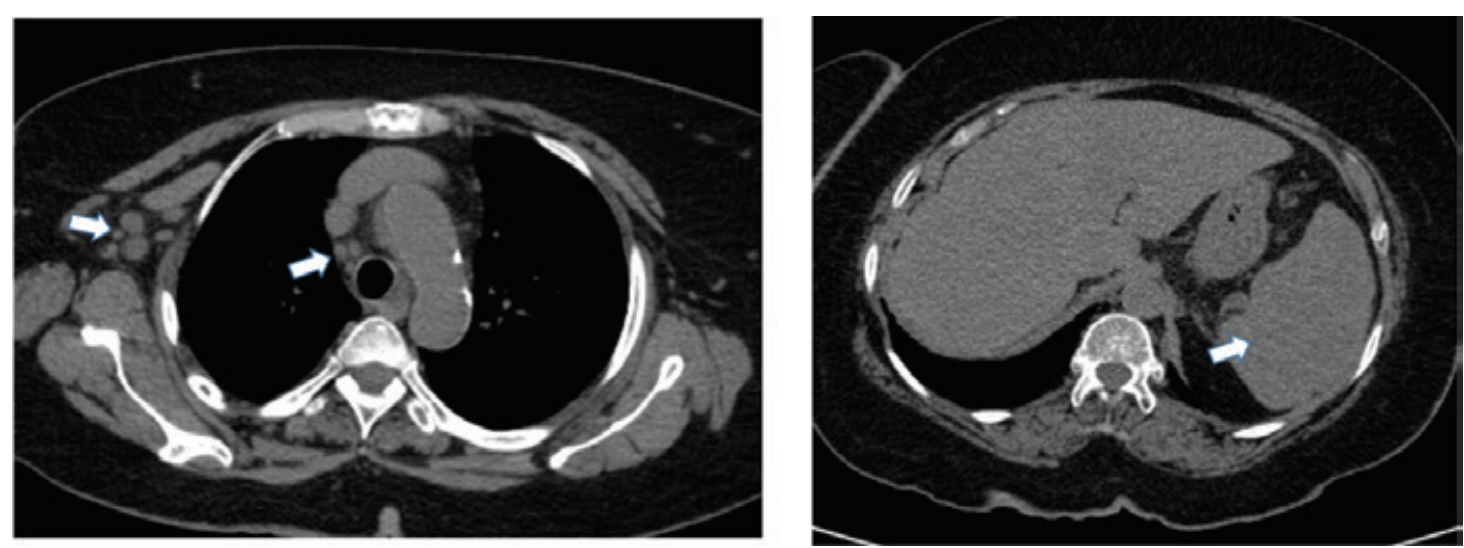

Figure 2: Mediastinal window shows mild mediastinal and right axillary lymphadenopathy. Also, mild enlargement of the spleen.
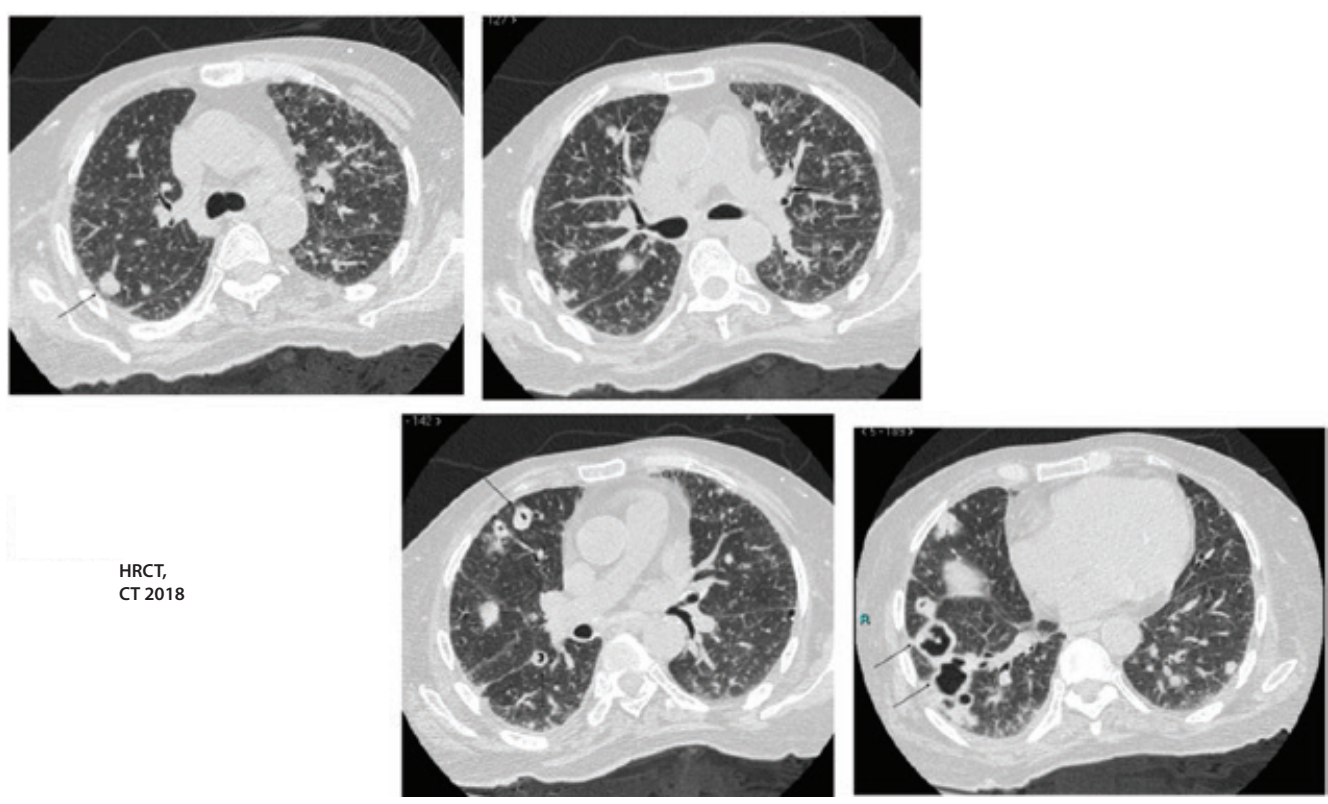

Figure 3: Follow up CT demonstrates a progressive enlargement of pulmonary nodules, few of which started to form central cavitations.
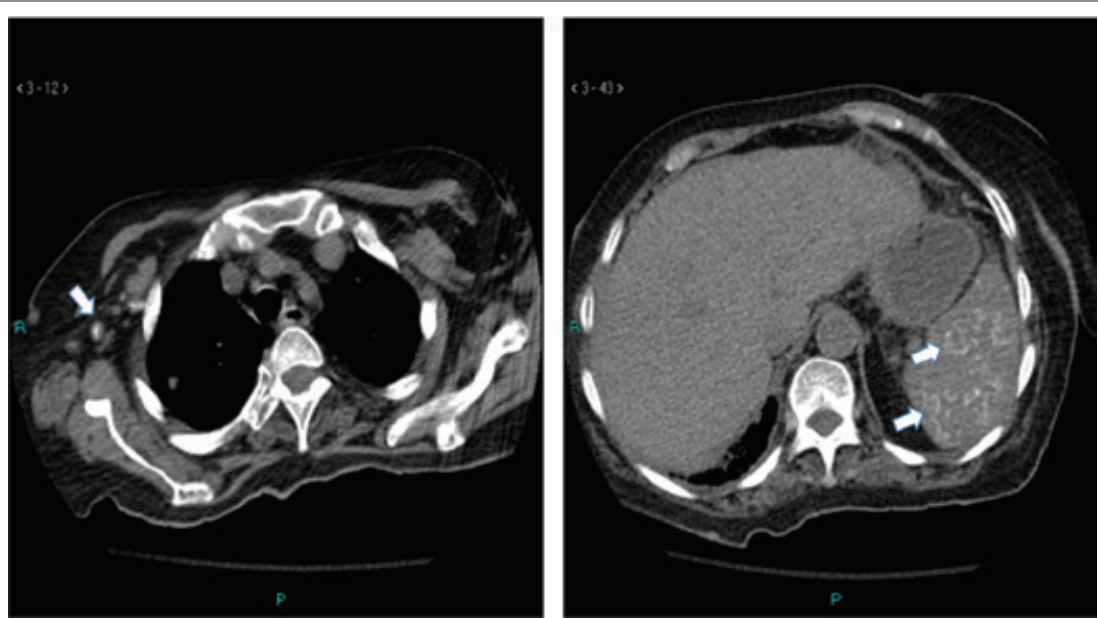

Figure 4: Mediastinal window reveals interval calcifications of the right axillary large lymph nodes and numerous foci of calcifications throughout the spleen. 

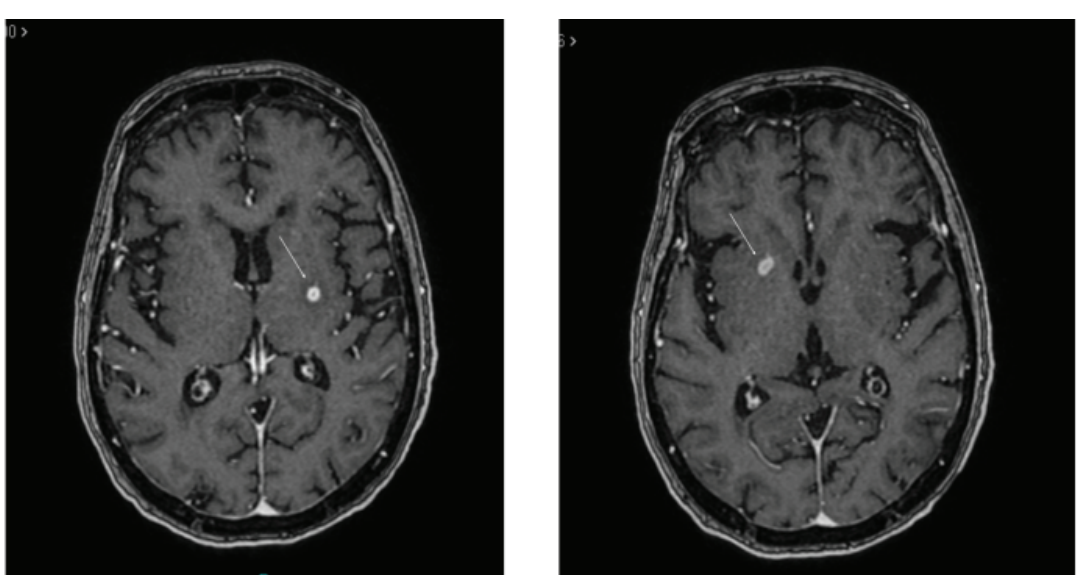

Figure 5: MRI brain shows ring enhancing lesions.

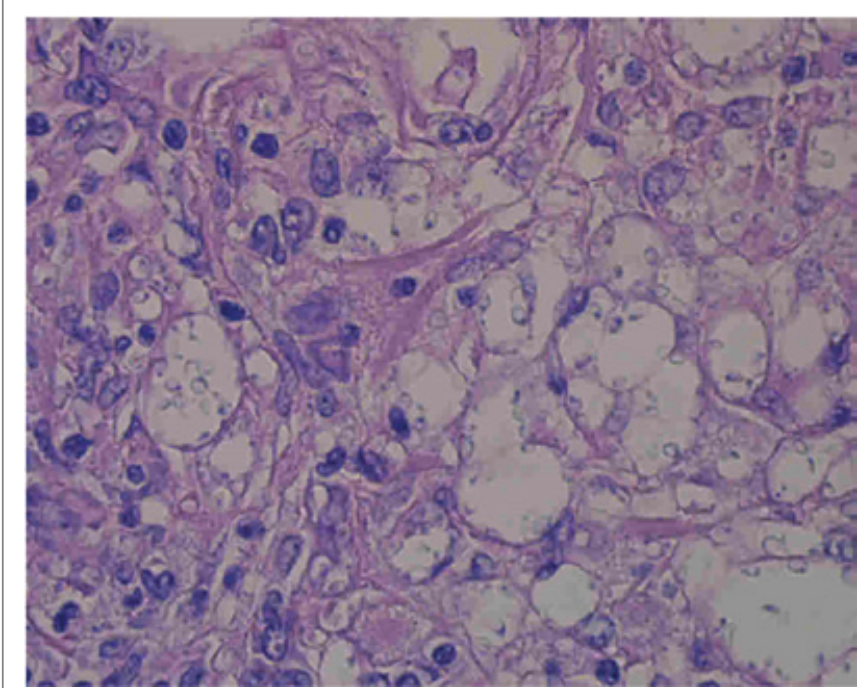

Figure 6: The Grocott methenamine silver stain reveals well fungal elements in black color $(\times 100)$.

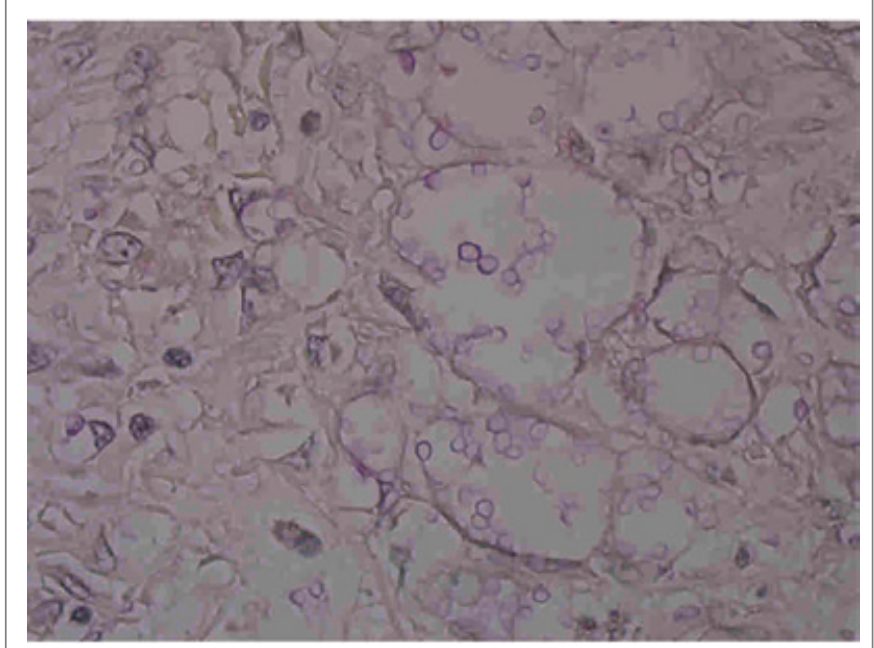

Figure 7: Thick mucinous bright red capsule with mucicarmine stain $(\times 100)$.

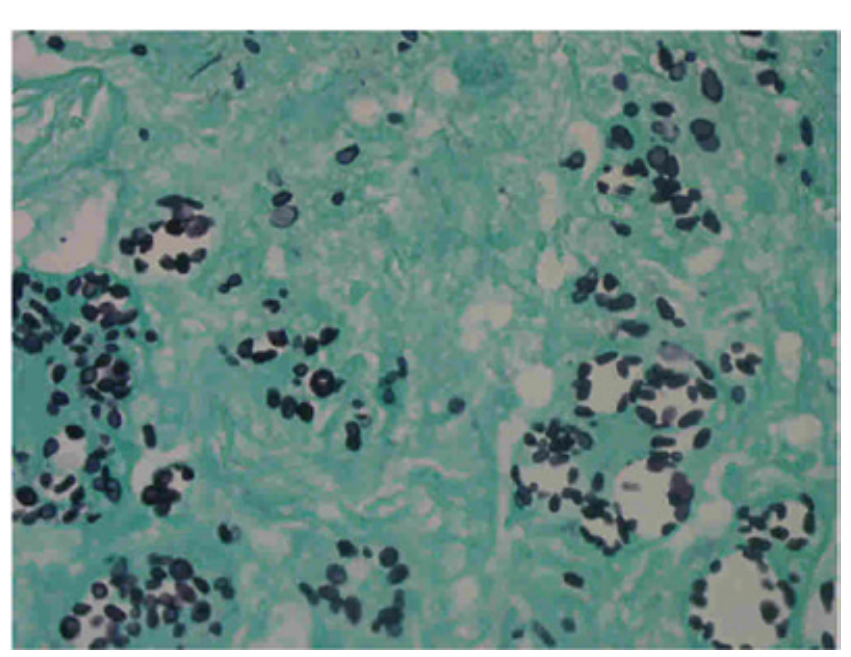

Figure 8: The Grocott methenamine silver stain reveals well fungal elements in black color $(\times 100)$.

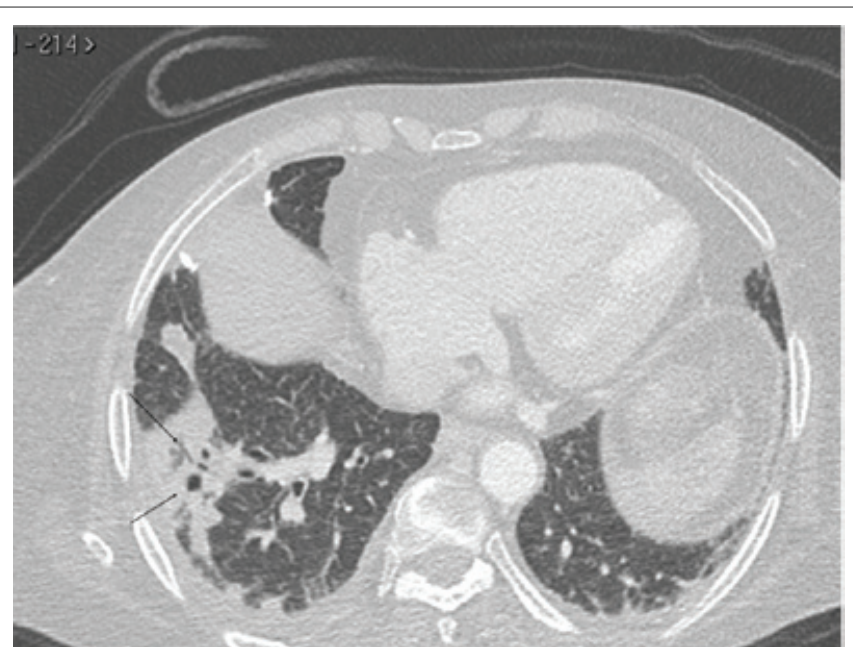

Figure 9: $\mathrm{CT}$ chest show marked improvement of the cavitating lung nodules. 
other CSF parameters were as follows: the opening pressure was normal, the total white blood cell count was of 2 , the protein level was of $0.473 \mathrm{~g} / \mathrm{l}(0.15-0.45)$, and the glucose level of $5.1 \mathrm{mmol} / \mathrm{l}(115-$ 130), with serum glucose $12.2 \mathrm{mmol} / \mathrm{l}$. serum Cryptococcal Ag 1:256 positive, but the results were negative from the CSF sample. Further testing included: HIV 1 and HIV 2 serologies, which were all negative. The patient CD4 counted $377 / \mathrm{Ml}$ (1000-2800), and the repeated one was $1200 / \mathrm{MI}$ (Table 1).

Interpretation of lymphocytes subsets: helper cell count unremarkable, suppressor cell count exceeding reference range. The low CD4/CD8-ratio is detectable in immunocompetent patients with systemic infections: virus, intracellular bacteria, or parasites.

Mycobacterium tuberculosis susceptibility isolate came to be fully sensitive to the four standard drugs. The patient was successfully treated with a combination therapy of anti-TB therapy and antifungal therapy. She improved clinically and radiologically as per figure 9 and 10 .

\section{Discussion and Conclusion}

Concomitant tuberculosis and cryptococcosis have rarely been reportedinHIV-infectedindividuals[3]. Theco-infectionoftuberculosis and cryptococcosis in immunocompetent individuals appears to be an even-rarer entity [4]. Important underlying diseases that are common to patients with pulmonary tuberculosis and cryptococcosis include immunodeficiency syndromes such as AIDS, kidney diseases, blood diseases, and cancer. Also, the proportion of such patients receiving corticosteroid treatment or immunosuppressive agents is high. There are several proposed mechanisms of immune deficiency in patients receiving anti-TNF therapy. TNF- $\alpha$ is essential for the formation and maintenance of granulomas [5,6]. As its inhibition can lead to increased risk of new tuberculosis infection, reactivation of latent tuberculosis, and can predispose to other granulomatous infections, such as Histoplasma capsulatum [7]. However, histoplasmosis is not endemic in Saudi Arabia [8]. A previous study that examined the soil of multiple cities in Saudi Arabia proved this [9]. TNF- $\alpha$ plays a role in macrophage activation and differentiation and phagosome formation and is critical for the clearance of intracellular pathogens (e.g., Listeria, Legionella, Salmonella) [5]. Subsequently, anti-TNF administration can predispose one to opportunistic infections such as Candida or Aspergillus because it causes neutropenia [10]. TNF- $\alpha$ is also crucial for immune responses against viral pathogens, and its inhibition could predispose to the Hepatitis B Virus (HBV) [11] or Varicella-Zoster Virus (VZV) [12]. C. neoformans and M. tuberculosis infections are believed to be acquired through inhalation of aerosolized particles from the environment. Primary pulmonary tuberculosis is thought to be a latent infection in many cases. Pulmonary tuberculosis in elderly patients may be etiologically associated with reactivation of a latent pulmonary infection. However, the mechanism of cryptococcosis onset is still unclear. Several possibilities have been considered, including primary progression, reactivation, and reinfection [13]. Our case appears to be one of the few reported

Table 1: Patient is considered immunocompetent based on the following findings.

\begin{tabular}{|l|c|c|}
\hline \multicolumn{1}{|c|}{ Parameter } & Results & Range \\
\hline Lymphocytes, absolute count & $2143 / \mathrm{ul}$ & $1000-2800$ \\
\hline T- lymphocytes (CD3+) & $1542 / \mathrm{ul}$ & $700-2100$ \\
\hline T- lymphocytes (CD3+) & $73 \%$ lymph & $55-83$ \\
\hline T - helper cells (CD3+/CD4+) & $434 / \mathrm{ul}$ & $300-1400$ \\
\hline T - helper cells (CD3+/CD4+) & $20-\%$ lymph & $28-57$ \\
\hline T - suppressor cells (CD3+/CD8+) & $1107+/ \mu l$ & $200-900$ \\
\hline T - suppressor cells (CD3+/CD8+) & $52+\% l y m p h$ & $10-39$ \\
\hline CD4+/CD8+ ratio & -0.39 & $1.0-3.6$ \\
\hline
\end{tabular}

Table 2: Reported case of co-infection tuberculosis and cryptococcosis in non-HIV patient without underlying diseases.

\begin{tabular}{|l|l|l|l|l|l|}
\hline \multicolumn{1}{|c|}{ Case/Ref } & Age/ Sex & \multicolumn{1}{|c|}{ Region } & \multicolumn{1}{c|}{$\begin{array}{c}\text { Pathological lesions ( } \\
\text { Tuberculosis/Cryptococosis) }\end{array}$} & \multicolumn{1}{|c|}{ Treatment Tuberculosis/Cryptococcosis) } & \multicolumn{1}{c|}{ Outcome } \\
\hline $1 /[3]$ & $61 / \mathrm{M}$ & United States & Lung/ CSF & INH, SM/AMPH-B & Recovered \\
\hline $2 /[4]$ & $69 / \mathrm{M}$ & United States & Lung/ Lung & INH, REP/KCZ & Recovered \\
\hline $3 /[5]$ & $51 / \mathrm{M}$ & Spain & CSF/CSF & INH, REP, EB, PZA/AMPH-B, 5-FC & Recovered \\
\hline $4 /[6]$ & $34 / \mathrm{F}$ & Saudi Arabia & Lymph node / vertebra & INH,REP, EB, PZA/FLCZ & Recovered \\
\hline $5 /[7]$ & $24 / \mathrm{F}$ & Italy & CSF/CSF & INH, REP, EB, PZA, SM/FLCZ, L-AMB & Recovered \\
\hline $6 /[8]$ & $18 / \mathrm{F}$ & Canada & Lung/ CSF, Lymph node & INH, REP, EB, PZA/AMPH-B, 5-FC, FLCZ & Recovered \\
\hline $7 /[9]$ & $65 / \mathrm{M}$ & India & Lung/ Lung & NA/AMPH-B, ITCZ & Recovered \\
\hline $8 /[10]$ & $58 / \mathrm{F}$ & Taiwan & Lymph node/ Lung & NA/FLCZ & Recovered \\
\hline $9 /[11]$ & $70 / \mathrm{M}$ & Iran & Lung/ CSF, Lung & INH, REP, EB, PZA/AMPH-B & Died \\
\hline $10 /[12]$ & $61 / \mathrm{M}$ & Rwanda & Lung, Bone marrow, Liver/ CSF & INH, REP, EB/AMPH-B, FLCZ & Recovered \\
\hline $11 /$ present case & $84 / F$ & Japan & $\begin{array}{l}\text { Lung, Bone marrow, Liver, skin/ } \\
\text { Lung }\end{array}$ & INH, REP, EB/FLCZ & Recovered \\
\hline
\end{tabular}



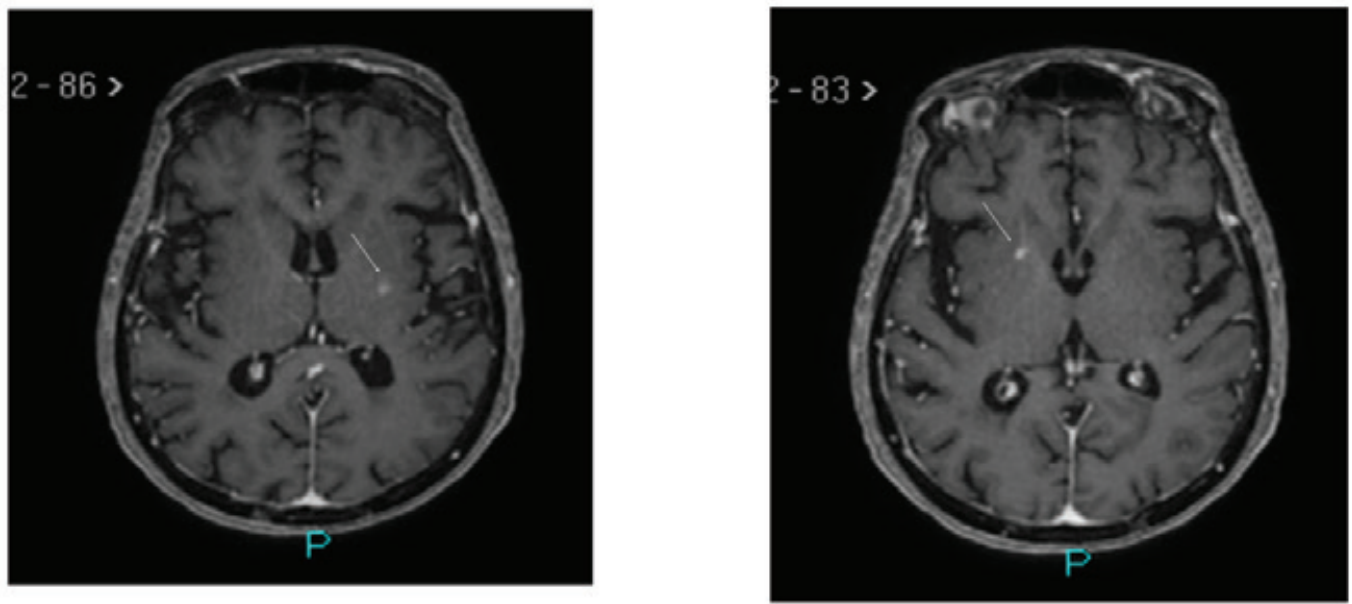

Figure 10: MRI brain demonstrates the interval decrease in the size of the previously detected ring enhancing lesions.

cases of co-infection with C. neoformans and M. tuberculosis as per table 2.

In the present case, it is impossible to know whether infection with tuberculosis preceded infection with Cryptococcus or vice versa. There is some evidence that both TB and Cryptococcus have immunomodulatory effects on host defenses. Three recent studies have explored the effects of TB on several different aspects of host immunity [14,15]. Two of them $[14,15]$ used cells that were isolated from bronchoalveolar lavage fluid to study the expression of immune mediators in patients with TB. The third study [16] used induced sputum samples from $\mathrm{TB}$ patients, patients with other lung diseases and healthy controls to do the same. Collectively, the results of these studies suggest that the expression of immunosuppressive mediators inhibits host defenses against TB. The immunosuppressive mediators that have been identified as being upregulated in patients with active TB include both intracellular (e.g., suppressors of cytokine signaling, and that associated with interleukin receptor-associated) and extracellular (interleukin [IL]-10, transforming growth factor-beta RII, IL-1Rn, IDO, and CD163) molecules $[14,15]$.

There is also evidence that C.neoformans can negatively affect the host immune system $[17,18]$. The main virulence factor of C.neoformans is an outer polysaccharide capsule that is composed primarily of glucuronoxylomannan (GXM) and galactoxylomannan (GalXM). GXM has been shown to have several effects on the host immune system, including inducing of suppressor $\mathrm{T}$ cells (which inhibit cell-mediated immunity), directly inhibiting $\mathrm{T}$ cell responses and inhibiting the movement of leukocytes into inflammatory sites [18]. A recent study demonstrated the ability of GXM to induce macrophage apoptosis in rats, both in vivo and in vitro [19]. This result was also documented in a study that used peritoneal macrophages to show that both GXM and GalXM can cause macrophage apoptosis [19].

\section{References}

1. Sawai T, Nakao T, Koga S, Ide S, Yoshioka S, et al. (2018) Miliary tuberculosis with co-existing pulmonary cryptococcosis in non-HIV patient without underlying diseases: a case report. BMC Pulm Med 18: 6 .
2. Van Tongeren L, Shaipanich T, Fleetham JA (2011) Coinfection with Cryptococcus gattii and Mycobacterium tuberculosis in an otherwise healthy 18-year-old woman. Can Respir J 18: e62-e63.

3. Rawat D, Capoor MR, Nair D, Deb M, Aggarwal P (2008) Concomitant TB and Cryptococcosis in HIV-infected Patients. Trop Doct 38: 251252.

4. Wang Q, Wang Z, Hao Y, Li W, Xin T, et al. (2018) Coinfection with cryptococcus and aspergillus in an immunocompetent adult: A case report. Medicine (Baltimore) 97: e12612.

5. Koo S, Marty FM, Baden LR (2010) Infectious complications associated with immunomodulating biologic agents. Infect Dis Clin North Am 24: 285-306.

6. Roach DR, Bean AG, Demangel C, France MP, Briscoe H, et al. (2002) TNF regulates chemokine induction essential for cell recruitment, granuloma formation, and clearance of mycobacterial infection. J Immunol 168: 4620-4627.

7. Wallis RS, Broder M, Wong J, Lee A, Hoq L (2005) Reactivation of latent granulomatous infections by infliximab. Clin Infect Dis 41: S194-S198.

8. Malak JA, Kurban AK, Bridi GS, Thaddeus JD (1969) Systemic fungus infections in northern Saudi Arabia: A survey of the occurrence of positive skin tests in 100 selected patients. Ann Trop Med Parasitol 63: 143-146.

9. Hedaithy AL, Abdulla SS (1983) Survey of Saudi Arabian soils for the etiologic agent of histoplasmosis. Thesis, Arizona State University.

10. Toruner M, Jr Loftus EV, Harmsen WS, Zinsmeister AR, Orenstein R, et al. (2008) Risk factors for opportunistic infections in patients with inflammatory bowel disease. Gastroenterology 134: 929-936.

11. Carroll MB, Forgione MA (2010) Use of tumor necrosis factor alpha inhibitors in hepatitis $B$ surface antigen-positive patients: a literature review and potential mechanisms of action. Clin Rheumatol 29: 1021-1029.

12. Strangfeld A, Listing J, Herzer P, Liebhaber A, Rockwitz K, et al. (2009) Risk of herpes zoster in patients with rheumatoid arthritis treated with anti-TNF-alpha agents. JAMA 301: 737-744.

13. Goldman DL, Khine H, Abadi J, Lindenberg DJ, La P, et al. (2001) Serologic evidence for Cryptococcus neoformans infection in early childhood. Pediatrics 107: E66. 
14. Bonecini-Almeida MG, Ho JL, Boéchat NL, Chitale $\mathrm{S}$, Doo $\mathrm{H}$ et al. (2004) Down-modulation of lung immune responses by interleukin-10 and transforming growth factor $\beta$ (TGF- $ß$ ) and analysis of TGF- $\beta$ receptors I and II in active tuberculosis. Infect Immun 72: 2628-2634.

15. Raju B, Hoshino Y, Belitskaya-Lévy I, Dawson R, Ress S, et al. (2008) Gene expression profiles of bronchoalveolar cells in pulmonary TB. Tuberculosis (Edinb) 88: 39-51.

16. Almeida AS, Lago PM, Boechat N, Huard RC, Lazzarini LCO, et al. (2009) Tuberculosis is associated with a down-modulatory lung immune response that impairs Th1-type immunity. J Immunol 183 718-731.
17. Ellerbroek PM, Walenkamp AM, Hoepelman Al, Coenjaerts FE (2004) Effects of the capsular polysaccharides of Cryptococcus neoformans on phagocyte migration and inflammatory mediators. Curr Med Chem 11: 253-266.

18. Villena SN, Pinheiro RO, Pinheiro CS, Nunes MP, Takiya CM, et al. (2008) Capsular polysaccharides galactoxylomannan and glucuronoxylomannan from Cryptococcus neoformans induce macrophage apoptosis mediated by Fas ligand. Cell Microbiol 10: 1274-1285.

19. Chiapello LS, Baronetti JL, Garro AP, Spesso MF, Masih DT (2008) Cryptococcus neoformans glucuronoxylomannan induces macrophage apoptosis mediated by nitric oxide in a caspaseindependent pathway. Int Immunol 20: 1527-1541. 